\title{
Species Distribution and Antifungal Susceptibility of Invasive Candidiasis: A 2016-2017 Multicenter Surveillance Study in Beijing, China
}

This article was published in the following Dove Press journal: Infection and Drug Resistance

\begin{abstract}
Li-Na Guo, ${ }^{1,2}$ Shu-Ying Yu, ${ }^{1-3}$ Meng Xiao, ${ }^{1,2}$ Chun-Xia Yang, ${ }^{4}$ Chun-Mei Bao, ${ }^{5}$ Yan-Hua Yu, ${ }^{6}$ Li-Yan Ye, ${ }^{7}$ Yang Yang, ${ }^{1,2}$ Ge Zhang, ${ }^{1,2}$ Jie Liu, ${ }^{8}$ Guo-Wei Liang, ${ }^{9}$ Rong Min, (iD) ${ }^{10}$ Yu Zhu, " Hong Lei, ${ }^{12}$ Yu-Lei Liu, ${ }^{13}$ Lin-Juan Liu, ${ }^{14}$ YunJian $\mathrm{Hu},{ }^{15}$ Po-Ren Hsueh, ${ }^{16}$ Ying-Chun $\mathrm{Xu}^{1,2}$

'Department of Clinical Laboratory, Peking Union Medical College Hospital, Chinese Academy of Medical Sciences, ${ }^{2}$ Beijing Key Laboratory for Mechanisms Research and Precision Diagnosis of Invasive Fungal Diseases (BZ0447), ${ }^{3}$ Graduate School, Peking Union Medical College, Chinese Academy of Medical Sciences, ${ }^{4}$ Department of Infectious Diseases \& Clinical Microbiology, Beijing Chao-Yang Hospital, Capital Medical University, ${ }^{5}$ Clinical Laboratory Medical Center, The Fifth

Medical Center of Chinese PLA General Hospital,

${ }^{6}$ Department of Clinical Laboratory, You'an Hospital, Capital Medical University, ${ }^{7}$ Center for

Clinical Laboratory Medicine, Chinese PLA General Hospital, ${ }^{8}$ Department of Clinical Laboratory, Seventh Medical Center, PLA General Hospital, ${ }^{9}$ Department of Clinical Laboratory, Aerospace Center Hospital, ${ }^{10}$ Department of Clinical Laboratory, Xuanwu Hospital, Capital Medical University, "'Department of Clinical Laboratory, National Cancer Center/National Clinical Research Center for Cancer/Cancer Hospital, Chinese Academy of Medical Sciences and Peking Union Medical College, ${ }^{12}$ Department of Clinical Laboratory, The 8th Medical Center of Chinese PLA General Hospital, ${ }^{13}$ Department of Clinical Laboratory, Beijing Anzhen Hospital, Capital Medical University, ${ }^{14}$ Department of Clinical Laboratory, Peking University Cancer Hospital and Institute, ${ }^{15}$ Department of Clinical Laboratory, Beijing Hospital, Beijing, People's Republic of China; ${ }^{16}$ Departments of Laboratory Medicine \& Internal Medicine, National Taiwan University Hospital, National Taiwan University College of Medicine, Taipei, Taiwan
\end{abstract}

Correspondence Ying-Chun Xu

Department of Clinical Laboratory, Peking Union Medical College Hospital, Chinese Academy of Medical Sciences, No. I, Shuaifuyuan Wangfujing, Dongcheng District, Beijing 100730, People's Republic of China

$\mathrm{Tel} / \mathrm{Fax}+861069159766$

Email xycpumch@।39.com

Po-Ren Hsueh

Departments of Laboratory Medicine \& Internal Medicine, National Taiwan University Hospital,

National Taiwan University College of Medicine,

Taipei, Taiwan

Tel +886-2-23224262 ext. 65355

Fax +886-2-23955072

Email hsporen@ntu.edu.tw
Objective: Invasive candidiasis (IC), a life-threatening fungal infection prevalent among hospitalized patients, has highly variable regional epidemiology. We conducted a multicenter surveillance study to investigate recent trends in species distribution and antifungal susceptibility patterns among IC-associated Candida spp. in Beijing, China, from 2016 to 2017.

Materials and Methods: A total of 1496 non-duplicate Candida isolates, recovered from blood and other sterile body fluids of IC patients, were identified using matrix-assisted laser desorption/ionization time of flight mass spectrometry combined with ribosomal DNA internal transcribed spacer (ITS) region sequencing. Broth microdilution-based susceptibility testing using six antifungal agents was also conducted.

Results: Candida albicans was the most frequently isolated species (49.9\%), followed by Candida tropicalis (15.5\%), Candida glabrata (14.7\%) and Candida parapsilosis (14.2\%). No significant differences in species distribution were observed when compared with a 2012-2013 dataset. Overall, the rates of susceptibility to fluconazole and voriconazole were high among C. albicans (98\% and 97.2\%, respectively) and C. parapsilosis species complex (91.1\% and $92 \%$, respectively) isolates but low among $C$. tropicalis $(81.5 \%$ and $81.1 \%$, respectively) isolates. In addition, the rate of azole resistance among $C$. tropicalis isolates increased significantly $(1.8$-fold, $P<0.05)$ compared with that observed in 2012-2013, while micafungin resistance rates were $<5 \%$ for all tested Candida species.

Conclusion: Our results suggest that species distribution has remained stable among ICassociated Candida isolates in Beijing. Resistance to micafungin was rare, but increased azole resistance among $C$. tropicalis isolates was noted. Our study provides information on local epidemiology that will be important for the selection of empirical antifungal agents and contributes to global assessments of antifungal resistance.

Keywords: invasive fungal infections, Candida, species distribution, antifungal resistance, surveillance, Beijing

\section{Introduction}

Invasive candidiasis (IC) has emerged as the most frequent fungal infection among immunocompromised patients and those hospitalized with serious underlying conditions. IC describes candidemia and other deep-seated candidiasis, with candidemia being the most common and life-threatening presentation. The global incidence of candidemia has increased notably over the past two decades, rising from 2 cases to 14 cases per 100,000 persons based on population-based studies. ${ }^{1-3}$ In addition, candidemia is associated with high mortality rates and long periods of hospitalization. For example, a PATH Alliance study in North America indicated that the crude 
12-week mortality rate of candidemia varied from $23.7 \%$ to $52.9 \%$, depending on the Candida species. ${ }^{4}$

The epidemiology of IC has significantly evolved in recent years. A progressive shift in the most common cause of infections from Candida albicans to non-albicans Candida spp. has been observed globally. ${ }^{5-7}$ Antifungal resistance is also an increasingly difficult challenge for the implementation of effective empirical and prophylactic strategies, ${ }^{8,9}$ with emerging species exhibiting resistance to multiple classes of antifungal agents. ${ }^{10}$ Both nationwide and local epidemiological surveillance studies are very important for detecting emerging resistance, and provide information for proper antifungal stewardship. In the present study, we investigated species distribution and antifungal susceptibility patterns from 2016 to 2017 in Beijing, China, and compared our findings with trends in IC epidemiology and resistance from a 2012-2013 dataset. $^{11}$

\section{Materials and Methods}

\section{Study Design}

A surveillance study was carried out from January 2016 to December 2017 and included 37 centers (24 university hospitals, six military hospitals, four tertiary general hospitals, two cancer hospitals, and one children's hospital) distributed across 12 districts in Beijing, China. The districts included Dongcheng (seven hospitals), Chaoyang (six hospitals), Haidian (six hospitals), Fengtai (five hospitals), Xicheng (four hospitals), Changping (two hospitals), Shijingshan (two hospitals), Fangshan (one hospital), Huairou (one hospital), Mentougou (one hospital), Pinggu (one hospital), and Tongzhou (one hospital). Consecutive non-duplicated Candida isolates retrieved from blood and other normally sterile body fluids were sent to the Department of Clinical Laboratory, Peking Union Medical College Hospital, for species confirmation and susceptibility testing. The isolates were stored at $-80^{\circ} \mathrm{C}$ until use. The study was approved by the Human Research Ethics Committee of Peking Union Medical College Hospital (No. S-K524).

\section{Species Identification}

Isolates were identified using matrix-assisted laser desorption/ionization time of flight mass spectrometry (MALDI-TOF MS; Vitek MS, bioMérieux, Marcy l'Etoile, France) analysis in combination with ribosomal DNA internal transcribed spacer (ITS) region sequencing, as previously described. ${ }^{12,13}$

\section{Antifungal Susceptibility Testing}

In vitro susceptibilities to fluconazole (FLC), voriconazole (VRC), itraconazole (ITC), 5-flucytosine (5-FC), amphotericin $\mathrm{B}$ (AMB), and micafungin (MCA) were tested according to the Clinical and Laboratory Standards Institute (CLSI) broth microdilution method (M27-A3). ${ }^{14}$ The minimum inhibitory concentration (MIC) of each reagent for each isolate was assessed after $24 \mathrm{~h}$ of incubation. Endpoints for 5-FC, MCA, and the azoles were set at a $50 \%$ reduction in growth relative to the drug-free control. For AMB, the MIC was defined as the lowest concentration at which there was no discernible growth. Quality control strains Candida krusei ATCC 6258 and Candida parapsilosis ATCC 22019 were included in each assay. Current species-specific clinical breakpoints (CBPs) or epidemiological cut-off values (ECVs) were used for interpretation of results. ${ }^{15,16}$ ECVs were used to define wild-type (WT) and non-wild-type (non-WT) isolates if no CBPs were available from the CLSI.

\section{Statistical Analysis}

All statistical analyses were performed using SPSS software version 24.0 (SPSS Inc., Chicago, IL, USA). Comparisons of continuous variables were performed using the chi-squared test or Fisher's exact test, as appropriate. A $P$-value of $<0.05$ was considered significant.

\section{Results \\ Species Distribution and Patient Characteristics}

From 2016 to 2017, 1496 non-duplicated Candida isolates comprising 26 Candida species were recovered from blood and other sterile body fluids from IC patients in Beijing (Table 1). Candida albicans was the most frequently isolated species (747 isolates, 49.9\%), followed by Candida tropicalis (15.5\%), Candida glabrata species complex (14.7\%), and Candida parapsilosis species complex (14.2\%). The median patient age was 57.8 years (range, 0-98 years), and the proportions of male and female patients were $59.3 \%$ and $40.7 \%$, respectively. Notably, isolation rates of most Candida species increased gradually with increasing age, with the highest isolation rates $(41.5 \%, 621 / 1496)$ observed among patients at the extreme end of the age spectrum ( $>65$ years). C. albicans was the predominant species across all age groups $>15$ years, whereas $C$. parapsilosis species complex were most common in infants (0-1 year). $C$ albicans accounted for approximately $39.4 \% \quad(218 / 554)$ of 
Table I Species Distribution of 1496 Invasive Candida Isolates Based on Clinical Characteristics

\begin{tabular}{|c|c|c|c|c|c|c|c|c|}
\hline \multirow[t]{2}{*}{ Characteristics } & \multicolumn{8}{|c|}{ No. of Isolates or Patients } \\
\hline & Total & $\begin{array}{l}\text { C. } \\
\text { albicans }\end{array}$ & $\begin{array}{l}\text { C. } \\
\text { tropicalis }\end{array}$ & $\begin{array}{l}\text { C. glabrata } \\
\text { complex }\end{array}$ & $\begin{array}{l}\text { C. parapsilosis } \\
\text { Complex }^{d}\end{array}$ & $\begin{array}{l}\text { C. } \\
\text { lusitaniae }\end{array}$ & $\begin{array}{l}\text { C. } \\
\text { krusei }\end{array}$ & $\begin{array}{l}\text { Other } \\
\text { Candida }^{\mathrm{e}}\end{array}$ \\
\hline Number of isolates & 1496 & 747 & 233 & 221 & 213 & 25 & 22 & 35 \\
\hline \multicolumn{9}{|l|}{ Patient age (years) } \\
\hline $0-1$ & 72 & 27 & 3 & 1 & 29 & 12 & 0 & 0 \\
\hline $2-14$ & 37 & 13 & 13 & 1 & 9 & I & 0 & 0 \\
\hline $15-49$ & 281 & 134 & 60 & 33 & 38 & 5 & 3 & 8 \\
\hline $50-65$ & 485 & 261 & 68 & 74 & 58 & 3 & 10 & 11 \\
\hline$>65$ & 621 & 312 & 89 & 112 & 79 & 4 & 9 & 16 \\
\hline \multicolumn{9}{|l|}{ Gender } \\
\hline Male & 887 & 412 & 143 & 114 & 169 & 14 & 17 & 18 \\
\hline Female & 609 & 335 & 90 & 107 & 44 & 11 & 5 & 17 \\
\hline \multicolumn{9}{|l|}{ Isolation site } \\
\hline Blood & 554 & 218 & 96 & 92 & 119 & 14 & 5 & 10 \\
\hline Ascitic fluid & 402 & 233 & 63 & 65 & 20 & 2 & 10 & 9 \\
\hline Pus & 133 & 70 & 20 & 12 & 26 & I & 0 & 4 \\
\hline Bile & 120 & 72 & 18 & 12 & 10 & 2 & 4 & 2 \\
\hline Bronchoalveolar lavage fluid & 102 & 62 & 12 & 16 & 3 & 4 & 2 & 3 \\
\hline Catheter & 82 & 37 & 11 & 12 & 18 & 1 & 1 & 2 \\
\hline Pleural fluid & 42 & 25 & 6 & 4 & 6 & 0 & 0 & 1 \\
\hline Tissue & 22 & 11 & 0 & 3 & 5 & 1 & 0 & 2 \\
\hline Cerebrospinal fluid & 15 & 8 & 4 & 0 & 3 & 0 & 0 & 0 \\
\hline Other specimens ${ }^{\mathrm{a}}$ & 24 & 11 & 3 & 5 & 3 & 0 & 0 & 2 \\
\hline \multicolumn{9}{|l|}{ Clinical department } \\
\hline ICU & 471 & 240 & 85 & 80 & 44 & 8 & 6 & 8 \\
\hline Surgery & 411 & 215 & 52 & 63 & 59 & 2 & 8 & 12 \\
\hline Medicine & 341 & 171 & 58 & 50 & 46 & 3 & 3 & 10 \\
\hline Emergency & 120 & 53 & 20 & 19 & 24 & 0 & 3 & I \\
\hline Other departments ${ }^{\mathrm{b}}$ & 153 & 68 & 18 & 9 & 40 & 12 & 2 & 4 \\
\hline
\end{tabular}

Notes: ${ }^{a}$ Includes joint fluid, vitreous humor, peritoneal dialysate fluid. ' Includes pediatrics, dermatology, gynecology and obstetrics, endocrinology, otorhinolaryngology. 'Includes Candida glabrata sensu stricto (2I5) and Candida nivariensis (6). Includes Candida parapsilosis sensu stricto (192), Candida metapsilosis (7), Candida orthopsilosis (I I) and

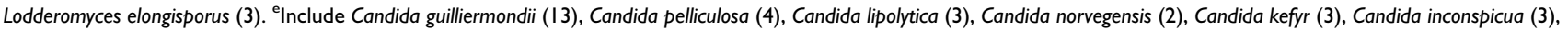
Candida haemulonii (2), Candida rugosa (I), Candida stellatoidea (I), Candida famata (I), Candida freyschussii (I), Candida fabianii (I).

bloodstream Candida isolates. C. parapsilosis species complex were the second most common bloodstream isolates, responsible for $21.5 \%$ of all cases. Of the 1496 IC cases, $31.5 \%(471 / 1496)$ occurred in intensive care units (ICUs) and $27.5 \%(411 / 1496)$ in surgical wards. However, C. albicans was the most frequently isolated species across the different clinical departments (Table 1).

\section{Antifungal Susceptibility}

Table 2 summarizes the results of in vitro susceptibility testing of the invasive Candida isolates recovered in this study. FLC and VRC were most active against C. albicans (98\% and 97.2\% susceptible, respectively) and
C. parapsilosis species complex $(91.1 \%$ and $92.0 \%$ susceptible, respectively). Overall, $94.1 \%$ of C. glabrata species complex isolates showed dose-dependent susceptibility to FLC, while decreased FLC susceptibility was observed among C. tropicalis ( $81.5 \%$ susceptible), C. guilliermondii (46.2\% non-WT) and C. lusitaniae (88\% non-WT) isolates. VRC was active against FLCresistant $C$. krusei isolates (90.9\% susceptible), but showed reduced activity against FLC-resistant $C$. tropicalis isolates (81.1\% susceptible), with $37 / 40(92.5 \%)$ FLC-resistant $C$. tropicalis isolates also showing VRC resistance. Notably though, all of the FLC-resistant $C$. tropicalis isolates were susceptible to MCA based on the 
Table 2 In vitro Susceptibilities of 1496 Candida Isolates to Six Antifungal Agents as Determined by the Broth Microdilution Method According to the Guidelines of the Clinical and Laboratory Standards Institute

\begin{tabular}{|c|c|c|c|c|c|c|c|c|c|}
\hline \multirow[t]{2}{*}{ Organism and Agent } & \multicolumn{3}{|c|}{ MIC (mg/L) ${ }^{b}$} & \multicolumn{4}{|c|}{$\begin{array}{l}\% \text { of Isolates in Each Indicated } \\
\text { Susceptibility Category by CBP }\end{array}$} & \multicolumn{2}{|c|}{$\begin{array}{l}\text { \% of Isolates by } \\
\text { ECV }^{\mathbf{d}}\end{array}$} \\
\hline & Range & $50 \%$ & $90 \%$ & $\mathbf{S}$ & S-DD & I & $\mathbf{R}$ & WT & non-WT \\
\hline $\begin{array}{l}\text { C. albicans }(\mathrm{n}=747) \\
\text { Fluconazole } \\
\text { Voriconazole } \\
\text { Itraconazole } \\
\text { Amphotericin B } \\
\text { 5-flucytosine } \\
\text { Micafungin }\end{array}$ & $\begin{array}{l}0.25-64 \\
0.008-2 \\
0.016-1 \\
0.016-2 \\
0.064-128 \\
0.008-2\end{array}$ & $\begin{array}{l}0.5 \\
0.016 \\
0.125 \\
0.5 \\
0.064 \\
0.064\end{array}$ & $\begin{array}{l}2 \\
0.064 \\
0.5 \\
1 \\
0.125 \\
0.125\end{array}$ & $\begin{array}{l}98 \\
97.2 \\
\\
\\
99.7\end{array}$ & 1.7 & 0 & $\begin{array}{l}0.3 \\
0.3 \\
\\
0.3\end{array}$ & 100 & 0 \\
\hline $\begin{array}{l}\text { C. tropicalis }(\mathrm{n}=233) \\
\text { Fluconazole } \\
\text { Voriconazole } \\
\text { Itraconazole } \\
\text { Amphotericin B } \\
\text { 5-Flucytosine } \\
\text { Micafungin }\end{array}$ & $\begin{array}{l}0.125-512 \\
0.008-32 \\
0.016-32 \\
0.25-2 \\
0.064-0.125 \\
0.008-2\end{array}$ & $\begin{array}{l}0.5 \\
0.032 \\
0.25 \\
1 \\
0.064 \\
0.032\end{array}$ & $\begin{array}{l}64 \\
2 \\
0.5 \\
1 \\
0.064 \\
2\end{array}$ & $\begin{array}{l}81.5 \\
81.1 \\
\\
\\
97.9\end{array}$ & 1.3 & $\begin{array}{l}3 \\
0.9\end{array}$ & $\begin{array}{c}17.2 \\
15.9 \\
\\
1.3\end{array}$ & $\begin{array}{l}96.6 \\
100\end{array}$ & $\begin{array}{l}3.4 \\
0\end{array}$ \\
\hline $\begin{array}{l}\text { C. glabrata species complex }(\mathrm{n}=22 \mathrm{I}) \\
\text { Fluconazole } \\
\text { Voriconazole } \\
\text { Itraconazole } \\
\text { Amphotericin B } \\
\text { 5-flucytosine } \\
\text { Micafungin }\end{array}$ & $\begin{array}{l}0.25-256 \\
0.016-16 \\
0.016-16 \\
0.008-2 \\
0.064-8 \\
0.008-4\end{array}$ & $\begin{array}{l}8 \\
0.125 \\
0.5 \\
1 \\
0.064 \\
0.032\end{array}$ & $\begin{array}{l}32 \\
0.5 \\
1 \\
1 \\
0.064 \\
0.064\end{array}$ & 91.9 & 94.1 & 5.9 & $\begin{array}{l}5.9 \\
\\
2.3\end{array}$ & $\begin{array}{c}82.4 \\
96.8 \\
100\end{array}$ & $\begin{array}{l}17.6 \\
3.2 \\
0\end{array}$ \\
\hline $\begin{array}{l}\text { C. parapsilosis species complex }(\mathrm{n}=2 \mid 3) \\
\text { Fluconazole } \\
\text { Voriconazole } \\
\text { Itraconazole } \\
\text { Amphotericin B } \\
\text { 5-flucytosine } \\
\text { Micafungin }\end{array}$ & $\begin{array}{l}0.25-128 \\
0.008-8 \\
0.016-2 \\
0.008-2 \\
0.064-64 \\
0.008-4\end{array}$ & $\begin{array}{l}1 \\
0.032 \\
0.25 \\
0.5 \\
0.064 \\
1\end{array}$ & $\begin{array}{l}2 \\
0.125 \\
0.5 \\
1 \\
0.125 \\
2\end{array}$ & $\begin{array}{l}91.1 \\
92 \\
\\
99.5\end{array}$ & 4.2 & $\begin{array}{l}4.7 \\
0.5\end{array}$ & $\begin{array}{l}4.7 \\
3.3 \\
\\
0\end{array}$ & 100 & 0 \\
\hline $\begin{array}{l}\text { C. lusitaniae }(n=25) \\
\text { Fluconazole } \\
\text { Voriconazole } \\
\text { Itraconazole } \\
\text { Amphotericin B } \\
\text { 5-flucytosine } \\
\text { Micafungin }\end{array}$ & $\begin{array}{l}0.25-16 \\
0.016-0.25 \\
0.064-0.5 \\
0.016-1 \\
0.064-2 \\
0.008-1\end{array}$ & $\begin{array}{l}0.5 \\
0.016 \\
0.25 \\
0.5 \\
0.064 \\
0.25\end{array}$ & $\begin{array}{l}2 \\
0.064 \\
0.5 \\
1 \\
0.25 \\
0.5\end{array}$ & & & & & $\begin{array}{l}88 \\
100 \\
92\end{array}$ & $\begin{array}{l}12 \\
0 \\
8\end{array}$ \\
\hline $\begin{array}{l}\text { C. krusei }(\mathrm{n}=22) \\
\text { Fluconazole } \\
\text { Voriconazole } \\
\text { Itraconazole } \\
\text { Amphotericin B } \\
\text { 5-flucytosine } \\
\text { Micafungin }\end{array}$ & $\begin{array}{l}8-512 \\
0.016-4 \\
0.032-2 \\
0.125-2 \\
0.064-8 \\
0.008-0.5\end{array}$ & $\begin{array}{l}16 \\
0.125 \\
0.5 \\
1 \\
2 \\
0.125\end{array}$ & $\begin{array}{l}32 \\
0.5 \\
0.5 \\
1 \\
4 \\
0.5\end{array}$ & $\begin{array}{l}90.9 \\
90.9\end{array}$ & & $\begin{array}{l}4.5 \\
9.1\end{array}$ & 4.5 & $\begin{array}{l}95.5 \\
100\end{array}$ & $\begin{array}{l}4.5 \\
0\end{array}$ \\
\hline $\begin{array}{l}\text { C. guilliermondii }(n=13) \\
\text { Fluconazole } \\
\text { Voriconazole }\end{array}$ & $\begin{array}{l}2-64 \\
0.064-2\end{array}$ & $\begin{array}{l}16 \\
0.25\end{array}$ & $\begin{array}{l}32 \\
1\end{array}$ & & & & & 46.2 & 53.8 \\
\hline
\end{tabular}

(Continued) 
Table 2 (Continued).

\begin{tabular}{|c|c|c|c|c|c|c|c|c|c|}
\hline \multirow[t]{2}{*}{ Organism and Agent } & \multicolumn{3}{|c|}{ MIC (mg/L) ${ }^{b}$} & \multicolumn{4}{|c|}{$\begin{array}{l}\text { \% of Isolates in Each Indicated } \\
\text { Susceptibility Category by CBP }\end{array}$} & \multicolumn{2}{|c|}{$\begin{array}{l}\text { \% of Isolates by } \\
\text { ECV }^{d}\end{array}$} \\
\hline & Range & $50 \%$ & $90 \%$ & $\mathbf{s}$ & S-DD & $\mathbf{I}$ & $\mathbf{R}$ & WT & non-WT \\
\hline Itraconazole & $0.064-2$ & 1 & 2 & & & & & & \\
\hline Amphotericin B & $0.25-2$ & 0.75 & 1 & & & & & & \\
\hline 5-flucytosine & $0.064-128$ & 0.064 & 4 & & & & & & \\
\hline Micafungin & $0.5-2$ & 1 & 2 & 100 & & 0 & 0 & & \\
\hline \multicolumn{10}{|c|}{ Other Candida species $(n=22)^{a}$} \\
\hline Fluconazole & $1-512$ & 8 & 32 & & & & & & \\
\hline Voriconazole & $0.016-16$ & 0.125 & 0.5 & & & & & & \\
\hline Itraconazole & $0.032-32$ & 0.5 & 1 & & & & & & \\
\hline Amphotericin B & $0.25-16$ & 0.5 & 1 & & & & & & \\
\hline 5-flucytosine & $0.064-128$ & 0.125 & 8 & & & & & & \\
\hline Micafungin & $0.008-2$ & 0.125 & 0.5 & & & & & & \\
\hline
\end{tabular}

Notes: ${ }^{2}$ Includes Candida pelliculosa $(n=4)$, Candida lipolytica $(n=3)$, Candida norvegensis $(n=2)$, Candida kefyr $(n=3)$, Candida inconspicua $(n=3)$, Candida haemulonii $(n=2)$, Candida rugosa $(n=1)$, Candida stellatoidea $(n=1)$, Candida famata $(n=1)$, Candida freyschussii $(n=1)$, Candida fabianii $(n=1)$. ${ }^{b} 50 \%$ and $90 \%$, MICs at which $50 \%$ and $90 \%$ of isolates are inhibited, respectively. ${ }^{\mathrm{C}} \mathrm{CBP}$, clinical breakpoint; S, susceptible; I, intermediate; S-DD, susceptible-dose dependent; R, resistant. ${ }^{\mathrm{d}} \mathrm{ECV}$, epidemiological cutoff values; WT, wild-type; non-WT, non-wild-type.

revised CBPs. As expected, MCA resistance was not prevalent, with only $0.3 \%(2 / 747)$ of $C$. albicans, $1.3 \%$ (3/233) of C. tropicalis, $2.3 \%(5 / 221)$ of $C$. glabrata species complex, and $4.5 \%(1 / 22)$ of $C$. krusei isolates demonstrating MCA resistance. For all Candida isolates, the MICs of AMB were $\leq 2 \mathrm{mg} / \mathrm{L}$, except for one $C$. haemulonii isolate for which the MIC was $16 \mathrm{mg} / \mathrm{L}$.

\section{Geographic Variation in Species Distribution and Susceptibility Profiles}

The four most prevalent species of Candida (C. albicans, C. glabrata species complex, C. tropicalis, and C. parapsilosis species complex) accounted for 94.5\% (1414/ 1496) of all isolates in the current study. Although these four species were predominant at each of the study hospitals, their frequencies and rank orders varied considerably among different hospitals in Beijing, as shown in Figure 1. For example, although $C$. albicans was the most prevalent species in samples from $27 / 37(73.0 \%)$ hospitals, the percentage at which it was isolated varied widely (from $0 \%$ to $100 \%$ ) among the different centers. Among the 10 hospitals where $C$. albicans was not the dominant species, $C$. parapsilosis species complex, C. glabrata species complex, or $C$. tropicalis was the most common species. Notably, 25 (1.67\%) isolates were identified as C. lusitaniae in the current study, representing $2.5 \%$ (14/554) of all bloodstream isolates. Ten of the 14 bloodstream C. lusitaniae isolates were recovered from a single hospital, clustered in samples from the neonatal ICUs (NICUs). In addition, significant variation in the detection rate of invasive Candida isolates was observed among the participating hospitals. Overall, $15 / 37$ (40.5\%) medical centers, each with 400-1200 hospital beds, produced fewer than 10 Candida isolates from routine clinical specimens (blood and other sterile body fluids) during the study period, which may be partly associated with the patient population and laboratory capacity. FLC susceptibility among $C$. tropicalis isolates was also highly variable, with some institutions reporting no azole resistance and others (JM, YD, and TR) reporting FLC resistance rates as high as $60-100 \%$. This may result from differences in clinician prescribing patterns associated with treatment of and prophylaxis against invasive candidiasis.

\section{Discussion}

A better understanding of the local epidemiology of IC and rates of antifungal resistance is critical for clinicians to make informed therapeutic decisions regarding antifungal prophylaxis for their patients while awaiting culture-based drug susceptibility data. ${ }^{17,18}$ To this end, we undertook a retrospective, laboratory-based, 2-year continuous IC surveillance study in Beijing.

IC has emerged as an important nosocomial infection, especially among critically ill patients hospitalized in the ICU. ${ }^{19}$ ICU patients may be particularly susceptible to such infections because they are exposed to broad-spectrum 


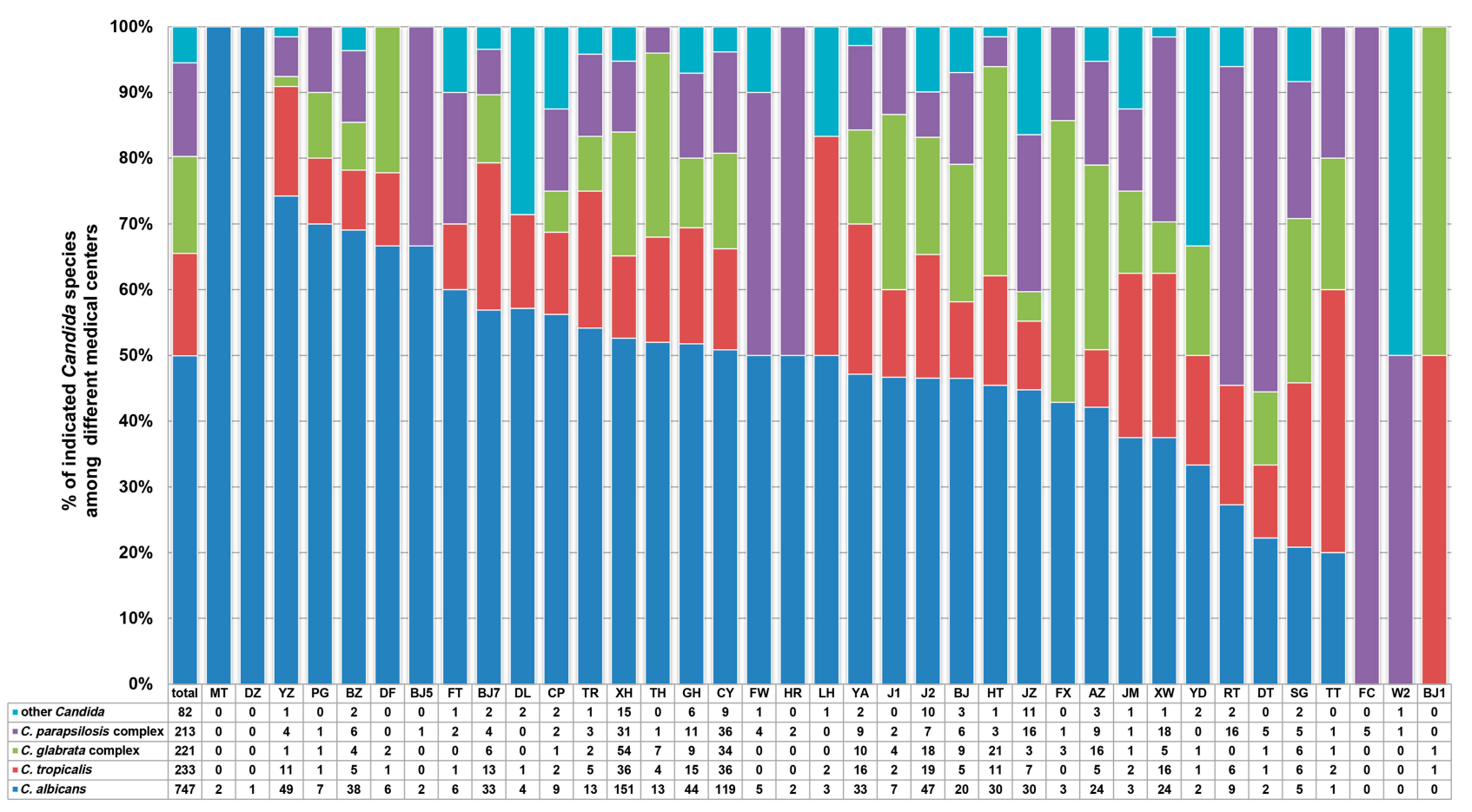

Figure I Geographic variations of C. albicans, C. tropicalis, C. glabrata complex, C. parapsilosis complex and other Candida species at 37 surveillance sites.

Abbreviations: MT, China Meitan General Hospital; DZ, Dongzhimen Hospital of Beijing University of Chinese Medicine; YZ, National Cancer Center/National Clinical Research Center for Cancer/Cancer Hospital; PG, Beijing Pinggu Hospital; BZ, Peking University Cancer Hospital and Institute; DF, Dongfang Hospital of Beijing University of Chinese Medicine; BJ5, Peking University Third Hospital; FT, Beijing Fengtai Hospital; BJ7, The 8th Medical Center of Chinese PLA General Hospital; DL, Beijing Electric Power Hospital; CP, Beijing Changping Hospital; TR, Beijing Tongren Hospital; XH, Peking Union Medical College Hospital; TH, Beijing Tsinghua Changgung hospital; GH, Chinese PLA General Hospital; CY, Beijing Chaoyang Hospital; FW, Fuwai hospital; HR, Beijing Huairou Hospital; LH, Beijing Luhe Hospital; YA, You'an Hospital; JI, The Fourth Medical Center of the Chinese PLA General Hospital; J2, The Fifth Medical Center of Chinese PLA General Hospital; BJ, Beijing Hospital; HT, Aerospace Center Hospital; JZ, The Seventh Medical Center of PLA General Hospital; FX, Fuxing Hospital; AZ, Anzhen Hospital; JM, Beijing Jingmei Group General Hospital; XW, Xuanwu Hospital; YD, Hebei Yanda Hospital; RT, Children's Hospital, Capital Institute of Pediatrics; DT, Beijing Ditan Hospital; SG, Peking University Shougang Hospital; TT, Beijing Tiantan Hospital; FC, Beijing Gynecology and Obstetrics Hospital; W2, Beijing Armed Police Corps Second Hospital; BJI, Beijing Chaoyang Hospital (West).

antibacterial agents, often have central venous catheters, have undergone major surgery, and are more likely to be elderly (>65 years) or experiencing immunosuppression. ${ }^{17}$ Importantly, a significant trend towards increased IC-associated mortality in ICU patients was observed. According to a large prospective hospital-based IC surveillance program in Paris, ${ }^{19}$ ICU patients had a higher overall death rate $(51 \%)$ than non-ICU patients $(30.7 \% ; P<0.001)$. Consistent with previous reports, ${ }^{9}$ samples from ICU patients contained the highest proportion of Candida isolates in our study (31.5\%). Furthermore, we noted that FLC resistance rates were much higher among $C$. tropicalis isolates from ICU patients than among isolates from other clinical wards $(24.7 \%$ versus $17.1 \%, P=0.021$ ), which may be an important consideration when developing guidelines for prophylaxis and treatment of IC patients.

Despite the global trend towards decreased frequency of $C$. albicans and increased frequency of non-albicans Candida species, ${ }^{5,6}$ C. albicans was the most commonly isolated Candida species at most hospitals in our study, accounting for $49.9 \%$ of all isolates. This prevalence rate is roughly consistent with data obtained in a study from Beijing in $2012-2013(52.5 \%),{ }^{11}$ and is similar to the prevalence in Europe $(52.5 \% ; 1997-2016$ SENTRY Program). However, it is higher than the prevalence rates reported in North America, Latin America, the AsiaPacific region and nationwide China (CHIF-NET), which range from $40 \%$ to $45 \%$. 6

Despite its overall prevalence, $C$. albicans accounted for only $39.4 \%(218 / 554)$ of the candidemia isolates with C. parapsilosis species complex identified as the leading non-albicans Candida bloodstream pathogen. C. parapsilosis species complex accounted for 21.5\% (119/554) of all bloodstream isolates, a prevalence rate that is similar to previous reports from China (CHIF-NET Program). ${ }^{9}$ In contrast, $C$. glabrata species complex were more prevalent among candidemia cases in the United States ${ }^{20}$ and some European countries. ${ }^{21}$ Interestingly, the species distribution of IC-associated pathogens shows regionality in Asia. A multi-center study in the Asia-Pacific region 
revealed that either C. tropicalis or C. parapsilosis species complex was the most prevalent non-albicans species isolated from bloodstream infections. ${ }^{22}$ In Pakistan, the prevalence of $C$. tropicalis surpassed that of $C$. albicans, ranking it as the most common IC-associated Candida species, followed by C. albicans in neonates and C. parapsilosis species complex in adults. ${ }^{23}$ In our study, $C$. tropicalis was the second and the third most prevalent cause of IC and candidaemia, respectively. It is thought that the trend towards increased prevalence of non-albicans species may be related in part to the increased use of FLC for treatment and prophylaxis. ${ }^{24}$

Of the five major Candida species associated with IC, C. krusei was the least frequently isolated, accounting for only $1-3 \%$ of isolates. ${ }^{21} \mathrm{C}$. krusei is a prominent pathogen among patients with hematologic malignancies and others who have received prolonged azole prophylaxis. ${ }^{17,25}$ Likewise, the frequency of C. krusei-associated IC infections was low in our study (1.5\%). Notably, there was a cluster of $C$. lusitaniae candidemia cases from a single NICU, emphasizing the importance of epidemiological studies and further investigations of the role of $C$. lusitaniae in neonatal fungemia. Similarly, a retrospective study from Kuwait shed light on the emerging role of $C$. lusitaniae as a healthcare-associated pathogen capable of causing fungemia in preterm neonates. ${ }^{26} \mathrm{C}$. lusitaniae is also recognized as a causative agent of breakthrough fungemia in cancer patients, ${ }^{27}$ with a study from the United States showing that the incidence of candidemia caused by $C$. lusitaniae has increased significantly over the past 16 years. $^{28}$

Candida auris, first reported in Japan in 2009, is an emerging pathogen that has quickly spread to multiple countries across several continents, becoming a significant clinical problem. ${ }^{10,29}$ C. auris has been associated with nosocomial outbreaks in ICUs, which is particularly concerning given that the large majority of isolates exhibit FLC resistance and variable susceptibility to other azoles, AMB, and echinocandins. ${ }^{10}$ In addition, C. auris can be misidentified by conventional commercial API-20C or Vitek-2 systems, necessitating the use of molecular methods or MALDI-TOF MS for identification. ${ }^{29}$ In the current study, all Candida isolates were forwarded to the central laboratory of the Department of Clinical Laboratory, Peking Union Medical College Hospital, for species confirmation by MALDI-TOF MS (Vitek MS, IVD Knowledgebase v.3.0 (C. auris included); bioMérieux), which was confirmed using ITS region sequencing.
Fortunately, none of the samples examined in the current study contained $C$. auris. Accurate identification of $C$. auris by MALDI-TOF MS is only reliable when the species is present in the database. Thus, laboratories should confirm with the manufacturer that the $C$. auris reference strain spectrum is present in their database. ${ }^{29}$

In our study, acquired resistance to FLC and VRC among C. albicans and C. parapsilosis species complex isolates was rare $(<5 \%)$. The rates of FLC resistance among C. glabrata species complex isolates decreased slightly between the study conducted in 2012-2013 $(7.2 \%)^{11}$ and the current study $(5.9 \%)(P=0.598)$. This may be caused by differences between participating hospitals in the two surveillance periods, with rates of resistance known to vary depending on patient population characteristics and by region or even hospital. ${ }^{1,2,5}$ However, rates of resistance to FLC among $C$. tropicalis isolates increased significantly, from $9.4 \%$ in $2012-2013^{11}$ to $17.2 \%$ in the current study period $(P<0.05)$. Thus, FLC resistance in $C$. tropicalis should be subject to careful monitoring. Furthermore, a high rate of cross-resistance (92.5\%) between FLC and VRC was observed among $C$. tropicalis isolates. Overall, our findings are in agreement with the national CHIF-NET study from China, which showed that the susceptibility of $C$. tropicalis to both FLC and VRC decreased continuously over the study period, dropping from $94.3 \%$ (for both azoles) in year one to about $76.8 \%$ in year five. ${ }^{9}$ High rates of FLC resistance among $C$. tropicalis isolates have also been recorded in the Asia-Pacific region $(18.1 \%)^{22}$ and Belgium (20\%). ${ }^{30}$ The association between increased resistance and widespread use of FLC prophylaxis and treatment in clinical practice has been widely debated and remains controversial. ${ }^{8,31}$ Regardless, increasing azole resistance calls for optimal FLC dosing and appropriate selection of antifungal agents for IC patients. Currently, the European Society of Clinical Microbiology and Infectious Diseases guidelines recommend the use of echinocandins as a first-line empirical treatment for IC prior to species identification and susceptibility testing. ${ }^{32}$ Worldwide, echinocandin resistance is rare and generally limited to $C$. glabrata isolates (resistance rates of $2-5 \%){ }^{1,6}$ The overall rate of echinocandin resistance was also very low in our study $(<5 \%)$; however, resistance rates of up to $12.3 \%$ have been noted in the United States. ${ }^{33}$ Therefore, with the increased use of echinocandins, it is important that we monitor rates of resistance. 
We observed a high degree of inter-laboratory variation in species distribution and detection rates of invasive Candida (Figure 1), which may be influenced by the patient population and laboratory diagnostic capacity of each center. A survey by the Asia Fungal Working Group $^{34}$ showed that the status of diagnostic mycology services in Asian countries is far from satisfactory. Approximately $31 \%$ of Chinese laboratories did not have a separate space for mycology diagnostics and most laboratories lacked continuing education and training in medical mycology. Furthermore, the National External Quality Assessment (EQA) program for mycology is still not available in China. With the increasing incidence of fungal disease, it is urgent that laboratories take steps to improve fungal diagnostics such as regular staff training and EQA program participation.

Our study had several limitations. First, this was a local epidemiological study, meaning that the results may not be generalizable to all patients with IC. However, data presented in our study may provide useful information for the treatment of fungal infections in areas outside of Beijing, as well as being used to establish better antifungal stewardship for IC patients in Beijing. Secondly, there were disparities among the numbers of isolates collected from different hospitals, which may influence the accuracy of the geographic picture of species distribution and antifungal resistance. Finally, we did not have access to FLC usage data, which would have allowed us to correlate FLC consumption and increased resistance rates.

\section{Conclusion}

Comparison of our results with data from 2012 to 2013 showed that species distribution of IC-associated Candida has remained stable in Beijing, and that acquired echinocandin resistance remains rare. Based on the increasing rate of azole resistance among $C$. tropicalis isolates, our study highlights the need for accurate species identification and azole susceptibility testing in clinical practice. In addition, our findings emphasize the importance of continued efforts to identify epidemiological changes associated with IC and determine the possible molecular mechanisms underlying azole resistance in C. tropicalis.

\section{Acknowledgments}

The co-principal investigators of the Beijing Invasive Fungal Surveillance Study Group are as follows: (1) Jian-Zhong Zhang, State Key Laboratory for Infectious Disease Prevention and Control, Collaborative Innovation Center for Diagnosis and Treatment of Infectious Diseases, National Institute for Communicable Disease Control and Prevention, Chinese Center for Disease Control and Prevention; (2) Hui-Zhu Wang, Beijing Ditan Hospital, Capital Medical University; (3) Wen-Jian Xu, Children's Hospital, Capital Institute of Pediatrics; (4) Jin-Qing Nong, Peking University Shougang Hospital; (5) Hai-Tong Gu, Beijing Tongren Hospital; (6) Li-jun Wang, Beijing Tsinghua Changgung Hospital; (7) Hai-Bin Wang, Fourth Medical Center of the Chinese PLA General Hospital; (8) Xiu-Juan He, Beijing Changping Hospital; (9) Fei-Yan Wang, Fuwai Hospital; (10) Xin Du, Beijing Fengtai Hospital; (11) Yan Li, Beijing Pinggu Hospital; (12) HaoChang Shou, Dongfang Hospital of Beijing University of Chinese Medicine; (13) Hui-Ying Jiang, Beijing Jingmei Group General Hospital; (14) Rui Zhao, Beijing Electric Power Hospital; (15) Shu-Mei Liu, Fu Xing Hospital, Capital Medical University; (16) Dian-Jun Wei, Hebei Yanda Hospital; (17) Fang Zhang, Beijing Luhe Hospital, Capital Medical University; (18) Fang-Qiang Li, Beijing Tiantan Hospital, Capital Medical University; (19) WenJuan Wang, Beijing Gynecology and Obstetrics Hospital, Capital Medical University; (20) Zhi-Zhong Xiao, Beijing Huairou Hospital; (21) Jia-Jia Zheng, Peking University Third Hospital; (22) Zhi-Gang Yue, China MeiTan General Hospital; (23) Jun Li, Beijing Armed Police Corps Second Hospital; (24) Jian-Mei Song, Beijing Chao-Yang Hospital, Capital Medical University; (25) Xi-Ming Yang, Dong zhi men Hospital of Beijing University of Chinese Medicine.

This work was supported by the Special Research Foundation for Capital Medical Development: Improvement of laboratory surveillance system and establishment of diagnostic service mode for invasive fungal diseases in Beijing (2016-1-4013); The National Natural Science Foundation of China $(81802049,81971979)$ and the Fundamental Research Funds for the Central Universities (3332020005). The funding agencies had no role in the study design, data collection and analysis, decision to publish, or preparation of the manuscript. We thank Tamsin Sheen, PhD, from Liwen Bianji, Edanz Editing China (www.en-author-services.edanzgroup.com), for editing the English text of a draft of this manuscript.

\section{Author Contributions}

Y-C X, L-N G, and MX conceived the work. S-Y Y, L-N $G, Y Y$, and GZ carried out species identification and antifungal susceptibility testing. L-N G, C-X Y, C-M B, MC, L-Y Y, JL, G-W L, RM, YZ, HL, Y-L L, L-J L, and Y-J H 
were responsible for data collection and analysis. L-N G, P-R H, and Y-C X drafted the manuscript. All authors contributed to data analysis, drafting, or revising the article, gave final approval of the version to be published, and agree to be accountable for all aspects of the work.

\section{Disclosure}

All authors declare that they have no conflicts of interest.

\section{References}

1. Kullberg BJ, Arendrup MC. Invasive candidiasis. $N$ Engl J Med. 2015;373(15):1445-1456. doi:10.1056/NEJMra1315399

2. Arendrup MC, Patterson TF. Multidrug-resistant Candida: epidemiology, molecular mechanisms, and treatment. J Infect Dis. 2017;216 (suppl_3):S445-S451. doi:10.1093/infdis/jix131

3. Liu WL, Lai CC, Li MC, et al. Clinical manifestations of candidemia caused by uncommon Candida species and antifungal susceptibility of the isolates in a regional hospital in Taiwan, 2007-2014. $J$ Microbiol Immunol Infect. 2019;52(4):612-619. doi:10.1016/j. jmii.2017.08.007

4. Horn David L, Neofytos D, Anaissie Elias J, et al. Epidemiology and outcomes of candidemia in 2019 patients: data from the prospective antifungal therapy alliance registry. Clin Infect Dis. 2009;48 (12):1695-1703. doi:10.1086/599039

5. Lamoth F, Lockhart SR, Berkow EL, Calandra T. Changes in the epidemiological landscape of invasive candidiasis. J Antimicrob Chemother. 2018;73(suppl_1):i4-i13. doi:10.1093/jac/dkx444

6. Pfaller MA, Diekema DJ, Turnidge JD, Castanheira M, Jones RN. Twenty Years of the SENTRY Antifungal Surveillance Program: results for Candida Species From 1997-2016. Open Forum Infect Dis. 2019;6(Suppl 1):S79-S94. doi:10.1093/ofid/ofy358

7. Tseng TY, Chen TC, Ho CM, et al. Clinical features, antifungal susceptibility, and outcome of Candida guilliermondii fungemia: an experience in a tertiary hospital in mid-Taiwan. J Microbiol Immunol Infect. 2018;51(4):552-558. doi:10.1016/j.jmii.2016.08.015

8. Fan X, Xiao M, Liao K, et al. Notable increasing trend in azole nonsusceptible candida tropicalis causing invasive candidiasis in China (August 2009 to July 2014): molecular epidemiology and clinical azole consumption. Front Microbiol. 2017;8:464. doi:10.3389/ fmicb.2017.00464

9. Xiao M, Sun ZY, Kang M, et al. Five-Year National Surveillance of Invasive Candidiasis: species Distribution and Azole Susceptibility from the China Hospital Invasive Fungal Surveillance Net (CHIFNET) Study. J Clin Microbiol. 2018;56(7):JCM.00577-00518. doi:10.1128/JCM.00577-18

10. Lockhart SR, Etienne KA, Vallabhaneni S, et al. Simultaneous emergence of multidrug-resistant Candida auris on 3 continents confirmed by whole-genome sequencing and epidemiological analyses. Clin Infect Dis. 2017;64(2):134-140. doi:10.1093/cid/ciw691

11. Guo LN, Xiao M, Cao B, et al. Epidemiology and antifungal susceptibilities of yeast isolates causing invasive infections across urban Beijing, China. Future Microbiol. 2017;12(12):1075-1086. doi:10.2217/fmb-2017-0036

12. Zhang L, Xiao M, Wang H, et al. Yeast identification algorithm based on use of the Vitek MS system selectively supplemented with ribosomal DNA sequencing: proposal of a reference assay for invasive fungal surveillance programs in China. J Clin Microbiol. 2014;52 (2):572-577. doi:10.1128/JCM.02543-13

13. Wang H, Li Y, Fan X, Chiueh TS, Xu YC, Hsueh PR. Evaluation of bruker biotyper and vitek MS for the identification of Candida tropicalis on different solid culture media. J Microbiol Immunol Infect. 2019;52(4):604-611. doi:10.1016/j.jmii.2017.11.002
14. Clinical and Laboratory Standards Institute. Reference Method for Broth Dilution Antifungal Susceptibility Testing of Yeasts. Approved Standard 3rd Ed. CLSI Document M27-A3. Wayne, PA: Humana Press; 2008.

15. Clinical and Laboratory Standards Institute. Reference Method for Broth Dilution Antifungal Susceptibility Testing of Yeasts. Fourth Informational Supplement M27-S4. Wayne, PA: Clinical and Laboratory Standards Institute; 2012.

16. Clinical and Laboratory Standards Institute. Performance Standards for Antifungal Susceptibility Testing of Yeasts. 1st Ed. CLSI Document M60. Wayne, PA; 2017.

17. McCarty TP, Pappas PG. Invasive Candidiasis. Infect Dis Clin North Am. 2016;30(1):103-124. doi:10.1016/j.idc.2015.10.013

18. Lin GL, Chang HH, Lu CY, et al. Clinical characteristics and outcome of invasive fungal infections in pediatric acute myeloid leukemia patients in a medical center in Taiwan. J Microbiol Immunol Infect. 2018;51(2):251-259. doi:10.1016/j.jmii.2016.08.011

19. Lortholary O, Renaudat C, Sitbon K, et al. Worrisome trends in incidence and mortality of candidemia in intensive care units (Paris area, 2002-2010). Intensive Care Med. 2014;40(9):1303-1312. doi:10.1007/s00134-014-3408-3

20. Webb BJ, Ferraro JP, Rea S, Kaufusi S, Goodman BE, Spalding J. Epidemiology and clinical features of invasive fungal infection in a US health care network. Open Forum Infect Dis. 2018;5(8):ofy187. doi:10.1093/ofid/ofy187

21. Hesstvedt L, Gaustad P, Andersen CT, et al. Twenty-two years of candidaemia surveillance: results from a Norwegian national study. Clin Microbiol Infect. 2015;21(10):938-945. doi:10.1016/j.cmi.2015. 06.008

22. Tan TY, Hsu LY, Alejandria MM, et al. Antifungal susceptibility of invasive Candida bloodstream isolates from the Asia-Pacific region. Med Mycol. 2016;54(5):471-477. doi:10.1093/mmy/myv114

23. Farooqi JQ, Jabeen $\mathrm{K}$, Saeed $\mathrm{N}$, et al. Invasive candidiasis in Pakistan: clinical characteristics, species distribution and antifungal susceptibility. J Med Microbiol. 2013;62(Pt 2):259-268. doi:10.1099/ jmm.0.048785-0

24. Arendrup MC, Dzajic E, Jensen RH, et al. Epidemiological changes with potential implication for antifungal prescription recommendations for fungaemia: data from a nationwide fungaemia surveillance programme. Clin Microbiol Infect. 2013;19(8):E343-E353. doi:10.1111/1469-0691.12212

25. Schuster MG, Meibohm A, Lloyd L, Strom B. Risk factors and outcomes of Candida krusei bloodstream infection: a matched, casecontrol study. $J$ Infect. 2013;66(3):278-284. doi:10.1016/j. jinf.2012.11.002

26. Khan Z, Ahmad S, Al-Sweih N, Khan S, Joseph L. Candida lusitaniae in Kuwait: prevalence, antifungal susceptibility and role in neonatal fungemia. PLoS One. 2019;14(3):e0213532. doi:10.1371/ journal.pone. 0213532

27. Minari A, Hachem R, Raad I. Candida lusitaniae: a cause of breakthrough fungemia in cancer patients. Clin Infect Dis. 2001;32(2):186190. doi: $10.1086 / 318473$

28. Jung DS, Farmakiotis D, Jiang Y, Tarrand JJ, Kontoyiannis DP. Uncommon Candida species fungemia among cancer patients, Houston, Texas, USA. Emerg Infect Dis. 2015;21(11):1942-1950. doi:10.3201/eid2111.150404

29. Jeffery-Smith A, Taori SK, Schelenz S, et al. Candida auris: a review of the literature. Clin Microbiol Rev. 2018;31(1).

30. Trouve C, Blot S, Hayette MP, et al. Epidemiology and reporting of candidaemia in Belgium: a multi-centre study. Eur J Clin Microbiol Infect Dis. 2017;36(4):649-655. doi:10.1007/s10096-016-2841-3

31. Shah DN, Yau R, Lasco TM, et al. Impact of prior inappropriate fluconazole dosing on isolation of fluconazole-nonsusceptible Candida species in hospitalized patients with candidemia. Antimicrob Agents Chemother. 2012;56(6):3239-3243. doi:10.1128/ AAC.00019-12 
32. Cornely OA, Bassetti M, Calandra T, et al. ESCMID* guideline for the diagnosis and management of Candida diseases 2012: non-neutropenic adult patients. Clin Microbiol Infect. 2012;18(Suppl 7):1937. doi:10.1111/1469-0691.12039

33. Alexander BD, Johnson MD, Pfeiffer CD, et al. Increasing echinocandin resistance in Candida glabrata: clinical failure correlates with presence of FKS mutations and elevated minimum inhibitory concentrations. Clin Infect Dis. 2013;56(12):1724-1732. doi:10.1093/ $\mathrm{cid} / \mathrm{cit} 136$
34. Chindamporn A, Chakrabarti A, Li R, et al. Survey of laboratory practices for diagnosis of fungal infection in seven Asian countries: an Asia Fungal Working Group (AFWG) initiative. Med Mycol. 2018;56(4):416-425. doi:10.1093/mmy/myx066

\section{Publish your work in this journal}

Infection and Drug Resistance is an international, peer-reviewed openaccess journal that focuses on the optimal treatment of infection (bacterial, fungal and viral) and the development and institution of preventive strategies to minimize the development and spread of resistance. The journal is specifically concerned with the epidemiology of antibiotic resistance and the mechanisms of resistance development and diffusion in both hospitals and the community. The manuscript management system is completely online and includes a very quick and fair peerreview system, which is all easy to use. Visit http://www.dovepress.com/ testimonials.php to read real quotes from published authors. 